\title{
The Crystal Structure of the Addition Compound between Diethylether and Monobromodichloromethane at $-130^{\circ} \mathrm{C}$
}

\author{
P. ANDERSEN and T. THURMANN-MOE \\ Universitetets Kjemiske Institutt, Blindern, Oslo 3, Norway
}

\begin{abstract}
$\left(\mathrm{C}_{2} \mathrm{H}_{5}\right)_{2} \mathrm{O}-\mathrm{HCBrCl}_{2}$ has been investigated by $\mathrm{X}$-ray methods at $-130^{\circ} \mathrm{C}$. Two crystal forms were found, both orthorhombic. One of these structures has been determined. The space group is $P n a 2_{1}$ and the cell dimensions are:

$a: 9.49 \pm 0.05 \AA, b: 14.12 \pm 0.07 \AA, c: 7.16 \pm 0.06 \AA$

with 4 molecules of each kind in the unit cell. The structure shows that the two molecules are linked together by a hydrogen bond between carbon and oxygen, the $\mathrm{C}-\mathrm{H}$...O distance being 3.10 $\AA$. The space group of the other form is either Pna2 or Pnam and the cell dimensions are $a: 16.89 \AA, b: 6.48 \AA, c: 10.15 \AA$ with four formula units in the cell.
\end{abstract}

Binary systems of diethyl ether and compounds of the type $\mathrm{HCX}_{1} \mathrm{X}_{2} \mathrm{X}_{3}$ $\mathbf{B}_{\left(\mathrm{X}_{1,2,3} \text { being halogen) have been investigated by different methods during }\right.}$ the last 30 years. Except in a few cases the conclusion has been drawn that association takes place between the molecules or that an addition compound is formed. All thermal measurements which have been carried out indicate that the compounds are associated, and a thermal analysis ${ }^{1}$ on the system $\left(\mathrm{C}_{2} \mathrm{H}_{5}\right)_{2} \mathrm{O}-\mathrm{HCCl}_{3}$ shows, beside a few unstable forms, a stable 1:1 compound having a m.p. $-91.6^{\circ} \mathrm{C}$. The heat of reaction of the same system ${ }^{2}$ at different temperatures has a maximum for ratio $1: 1$, at $24^{\circ} \mathrm{C}, c a .1150 \mathrm{cal} / \mathrm{mole}$. Dipole moment measurements of the system in benzene ${ }^{3}$ shows that a complex is established even at low concentrations. Two infrared investigations ${ }^{4,5}$ lead to the conclusion that a hydrogen bond is formed, a fairly strong one in the case of chloroform and a less stable one in the case of bromoform. Another infrared ${ }^{6}$ and a Raman ${ }^{7}$ investigation could not, however, give conclusive evidence regarding the complex formation. Diffusion ${ }^{8}$ and ultra-sound ${ }^{9}$ measurements indicate an assocition, however. The system ether-chloroform was discussed at a Faraday Society meeting $1936 .^{10}$

\section{EXPERIMENTAL}

In order to utilize the advantage of a heavy atom, bromodichloromethane was preferred to chloroform. By weighing at low temperature, an equimolecular mixture of this compound and absolute ether was obtained.

Acta Chem. Scand. 18 (1964) No. 2 
The melting point of the mixture was determined with a copper-constantan thermocouple, by slow heating and cooling. The temperature-time diagram of both freezing and melting showed a m.p. of $-94^{\circ} \mathrm{C}$ for the $1: 1$ compound $\left(\mathrm{C}_{2} \mathrm{H}_{5}\right)_{2} \mathrm{O}-\mathrm{HCBrCl}_{2}$.

The crystals were grown in capillary tubes of $0.2 \mathrm{~mm}$ diameter and the growth was studied under a polarizing microscope. If precautions were taken to remove all impurities both from the capillary tube and the liquid, serious undercooling made it impossible to grow single crystals. Traces of paper fibres made the undercooling negligible, however. The compound showed a marked tendency to form thin needles, and single crystals were obtained only in rare cases. In one case an uncontrolled sudden growth from the "warm" end of the tube gave a large single crystal different from those obtained by the usual growth from the "cold" end. Because of the poor quality of this crystal the X-ray diagrams obtained from it were not well suited for a structure determination. Attempts were made to reproduce this type of crystal but unfortunately without success. Nevertheless a structure determination based on the diffraction diagram obtained is now being attempted.

\section{CRYSTALLOGRAPHIC DATA}

The X-ray data were obtained from oscillation and Weissenberg photographs. Owing to the construction of the cooling system the higher layer lines had to be recorded using the "normal beam" method. Unfiltered CuKa radiation and the multiple-film technique were employed. The intensities were estimated visually by comparison with an intensity scale and then corrected in the usual way to obtain $\left|F_{0}\right|$ values.

The two types of crystals (I and II) obtained as mentioned above were both orthorhombic with unit cells having the dimensions:

I $a: 16.89 \AA, b: 6.48 \AA, c: 10.15 \AA$

II $a: 9.49 \pm 0.05 \AA, b: 14.12 \pm 0.07 \AA, c: 7.16 \pm 0.06 \AA$.

Four molecules of each kind in the unit cell correspond to the densities $d_{\mathrm{r}}: 1.42 \mathrm{~g} / \mathrm{cm}^{3}$ and $d_{\mathrm{Ir}}: 1.65 \mathrm{~g} / \mathrm{cm}^{3}$ which are of the expected order of magnitude. As mentioned above a crystal of type I was obtained only once. So far only the crystal structure of type II has been determined.

The systematic extinctions were the same for both types of crystals. In the $h 0 l$ zone reflexions are present when $h=2 n$ and in the $0 k l$ zone when $k+l=2 n$ and the space group could thus be either Pnam or Pna2 $2_{1}$. The compound might very well possess a mirror plane but not a center of symmetry; with 4 molecules in the unit cell either space group is thus possible.

\section{THE STRUCTURE DETERMINATION}

From the Patterson projection $P(u v)$ it was possible to determine the halogen parameters fairly accurately. In order to interpret the major peaks one had to assume an overlap of the two chlorine atoms in this projection. All peaks which might contain halogen-halogen vectors were explained by these parameters. If Pnam were the correct space group this overlap should be exact.

A Fourier projection based on the signs calculated from the halogen parameters did not give definite indications as to the position of the ether molecule. In the case of the space group Pnam a projection along the $c$-axis represents a projection onto a mirror plane. Whether the complex formation depends on a charge transfer bond ${ }^{11}$ where the ether oxygen acts as the electron donor 
Table 1. Atomic coordinates and standard deviatons.

$\begin{array}{cccccccc} & x & y & z & \sigma(x) & \sigma(y) & \sigma(z) & B \\ \mathrm{Br} & 0.1763 & 0.2276 & 0.000 & 0.0006 & 0.0004 & & 2.6 \\ \mathrm{Cl} & 0.391 & 0.113 & 0.213 & 0.002 & 0.001 & 0.009 & 1.9 \\ \mathrm{Cl} & 0.391 & 0.112 & -0.213 & 0.002 & 0.001 & 0.009 & 1.9 \\ \mathrm{C}_{1} & 0.273 & 0.115 & 0.000 & 0.010 & 0.006 & & 1.8 \\ \mathrm{C}_{2} & 0.288 & -0.152 & 0.000 & 0.005 & 0.004 & & 2.0 \\ \mathrm{C}_{3} & 0.127 & -0.152 & 0.050 & 0.006 & 0.004 & & 1.7 \\ \mathrm{O}^{2} & 0.084 & -0.060 & 0.000 & 0.006 & 0.002 & & 2.4 \\ \mathrm{C}_{4} & -0.071 & -0.057 & 0.050 & 0.006 & 0.004 & & 1.0 \\ \mathrm{C}_{5} & -0.119 & 0.055 & 0.000 & 0.005 & 0.004 & & \end{array}$

and bromine as the acceptor or on a hydrogen bond, a mirror plane is equally possible. The plane of the ether molecule might be either perpendicular to the mirror plane or coincide with it. In the latter case the three bonds from the oxygen atom would all have to be in the mirror plane, a possibility which does not, however, appear probable. Only a refinement assuming the space group $P n a 2_{1}$ can lead to a decision whether this or the more symmetric space group is the correct one. A trial computation assuming the space group Pnam and the plane of the ether molecule perpendicular to the mirror plane did not bring the $R$ factor below 0.24 which was the value obtained by including only the halogens. A difference synthesis $\left(F_{0}-F_{\text {c.hal. }}\right)$ gave strong indications for placing the atoms of the ether molecule in the plane of projection. As far as the monobromodichloromethane molecule is concerned the difference map showed that the two chlorine atoms overlapped exactly and a resolved peak appeared for the central carbon atom. Successive Fourier refinements including all the lighter atoms finally brought the $R$ factor down to 0.13 using an isotropic temperature factor $B=2.2 \AA^{2}$ for all atoms. A least squares refinement based on all zero and non-zero observed reflexions, of the $x$ - and $y$-parameters of all atoms and the $z$-parameters of the chlorine atoms resulted in small changes in some of the parameters. The main orientation of the complex was not altered by the refinement. As only the $h k 1$ and $h k 2$ reflexions were obtained and the inner part of these layer lines were missing due to the use of the normal beam method, and also because of the poor quality of these layer line reflexions, the $z$-parameters could not be determined very accurately. The parameters (and their standard deviations) deduced from the least squares refinement and also the $z$-parameters based on trial and error calculations are listed in Table 1. The refinement was based on 322 zero and non-zero observed reflexions and on isotropic temperature factors. The final $R$-value was about $19 \%$. The parameters obtained from the projection and from the least squares refinement both gave approximately the same $R$-value of about $17 \%$ for the non-zero reflexions.

Fig. 1 shows the Fourier projection along the $c$-axis. The ether and monobromodichloromethane molecules are indicated by straight lines between the atomic positions determined from this projection. 
Table 2. Observed and calculated structure factors and phase angles.

\begin{tabular}{|c|c|c|c|c|c|c|c|c|c|c|}
\hline$h$ & $k$ & $l$ & $\left|F_{\mathrm{o}}\right|$ & $\left|F_{\mathrm{c}}\right|$ & $\alpha^{\circ}$ & $h$ & $k$ & $\left|F_{\mathrm{o}}\right|$ & $\left|F_{\mathrm{c}}\right|$ & $a^{\circ}$ \\
\hline 2 & 0 & 0 & 59 & 57 & 180 & 3 & & 86 & 70 & 0 \\
\hline 4 & & & 138 & 126 & 180 & 4 & & 45 & 43 & 0 \\
\hline 6 & & & $<16$ & 8 & 0 & 5 & & 24 & 15 & 0 \\
\hline 8 & & & $<18$ & 13 & 180 & 6 & & $<18$ & 10 & 180 \\
\hline 10 & & & 34 & 42 & 0 & 7 & & 76 & 77 & 180 \\
\hline 1 & 1 & & 13 & 7 & 180 & 8 & & $<18$ & 17 & 0 \\
\hline 2 & & & 27 & i & 0 & 9 & & $<18$ & 4 & 0 \\
\hline 3 & & & 34 & 21 & 0 & l & 7 & 66 & 68 & 180 \\
\hline 4 & & & 124 & 111 & 0 & 2 & & 28 & 22 & 180 \\
\hline 5 & & & 22 & 13 & 0 & 3 & & 74 & 71 & 0 \\
\hline 6 & & & 72 & 65 & 180 & 4 & & 57 & 46 & 180 \\
\hline 7 & & & $<13$ & 3 & 180 & 5 & & 25 & 19 & 180 \\
\hline 8 & & & 45 & 46 & 180 & 6 & & 40 & 32 & 0 \\
\hline 9 & & & 42 & 34 & 180 & 7 & & $<18$ & 14 & 0 \\
\hline 10 & & & 44 & 45 & 0 & 8 & & 44 & 39 & 0 \\
\hline 0 & 2 & & 33 & 66 & 180 & 9 & & $<18$ & 12 & 0 \\
\hline 1 & & & 83 & 80 & 180 & 0 & 8 & 75 & 63 & 0 \\
\hline 2 & & & 92 & 95 & 0 & 1 & & 112 & 101 & 0 \\
\hline 3 & & & 79 & 52 & 180 & 2 & & 53 & 28 & 180 \\
\hline 4 & & & $<11$ & 3 & 180 & 3 & & 39 & 18 & 0 \\
\hline 5 & & & 21 & 25 & 0 & 4 & & 37 & 32 & 180 \\
\hline 6 & & & 82 & 78 & 180 & 5 & & 45 & 44 & 180 \\
\hline 7 & & & 48 & 21 & 0 & 6 & & $<18$ & 13 & 0 \\
\hline 8 & & & 40 & 38 & 0 & 7 & & $<20$ & 10 & 0 \\
\hline 9 & & & $<18$ & 15 & 0 & 8 & & $<18$ & 7 & 0 \\
\hline 1 & 3 & & 54 & 42 & 0 & 9 & & $<16$ & 15 & 180 \\
\hline 2 & & & 167 & 195 & 0 & 1 & 9 & 32 & 44 & 180 \\
\hline 3 & & & $<11$ & 10 & 0 & 2 & & $<16$ & 7 & 180 \\
\hline 4 & & & 32 & 50 & 180 & $\overline{3}$ & & 30 & 22 & 180 \\
\hline 5 & & & 51 & 46 & 180 & 4 & & $<18$ & 17 & 0 \\
\hline 6 & & & 37 & 26 & 180 & 5 & & 92 & 94 & 0 \\
\hline 7 & & & 24 & 18 & 180 & 6 & & $<18$ & 13 & 180 \\
\hline 8 & & & 25 & 12 & 180 & 7 & & & 6 & 0 \\
\hline 9 & & & 37 & 36 & 0 & 8 & & $<18$ & 4 & 180 \\
\hline 0 & 4 & & 80 & 39 & 180 & 9 & & 49 & $5 \overline{2}$ & 180 \\
\hline 1 & & & 17 & 30 & 0 & 0 & 10 & $<16$ & 4 & 180 \\
\hline 2 & & & 51 & 46 & 180 & 1 & & 84 & 86 & 180 \\
\hline 3 & & & 22 & 11 & 180 & 2 & & 25 & 8 & 0 \\
\hline 4 & & & 52 & 39 & 0 & 3 & & 21 & 24 & 180 \\
\hline 5 & & & 24 & 22 & 180 & 4 & & 28 & 14 & 180 \\
\hline 6 & & & 83 & 74 & 0 & 5 & & 37 & 38 & 0 \\
\hline 7 & & & 38 & 33 & 0 & 6 & & $<18$ & 11 & 180 \\
\hline 8 & & & 60 & 56 & 180 & 7 & & $<18$ & 6 & 180 \\
\hline 9 & & & $<18$ & 9 & 180 & 8 & & $<18$ & 16 & 0 \\
\hline 1 & 5 & & 91 & 83 & 0 & 1 & 11 & 47 & 35 & 180 \\
\hline 2 & & & 74 & 80 & 180 & 2 & & 45 & 40 & 0 \\
\hline 3 & & & 107 & 93 & 180 & 3 & & 53 & 47 & 0 \\
\hline 4 & & & 50 & 48 & 0 & 4 & & $<18$ & 5 & 0 \\
\hline 5 & & & 29 & 8 & 180 & 5 & & $<18$ & 15 & 180 \\
\hline 6 & & & $<18$ & 2 & 0 & 6 & & $<18$ & 13 & 180 \\
\hline 7 & & & $<18$ & 7 & 0 & 7 & & $<16$ & 4 & 180 \\
\hline 8 & & & $<20$ & 13 & 180 & 8 & & 29 & 23 & 180 \\
\hline 9 & & & $<18$ & 15 & 0 & 0 & 12 & 37 & 37 & 180 \\
\hline 0 & 6 & 0 & 132 & 105 & 180 & 1 & & $<18$ & 3 & 0 \\
\hline 1 & & & $<11$ & 6 & 180 & 2 & & $<18$ & 2 & 180 \\
\hline 2 & & & $<13$ & 10 & 0 & 3 & & 45 & 40 & 180 \\
\hline
\end{tabular}

Acta Chem. Scand. 18 (1964) No. 2 


\begin{tabular}{|c|c|c|c|c|c|c|c|c|c|c|}
\hline$h$ & $k$ & $l$ & $\left|\boldsymbol{F}_{\mathrm{o}}\right|$ & $\left|F_{c}\right|$ & $a^{\circ}$ & $h$ & $k$ & $\left|F_{\mathrm{o}}\right|$ & $\left|F_{\mathrm{c}}\right|$ & $a^{\circ}$ \\
\hline 4 & & & 31 & 24 & 0 & 7 & & $<30$ & 21 & 98 \\
\hline 5 & & & $<18$ & 12 & 180 & 8 & & $<33$ & 6 & 90 \\
\hline 6 & & & $<16$ & 9 & 0 & 0 & 5 & 73 & 68 & 90 \\
\hline 7 & & & 50 & 56 & 0 & 1 & & 24 & 35 & 91 \\
\hline 1 & 13 & & 48 & 53 & 0 & 2 & & 81 & 64 & -88 \\
\hline 2 & & & $<18$ & 9 & 0 & 3 & & 28 & 20 & \\
\hline 3 & & & 59 & 55 & 180 & 4 & & $<23$ & 15 & \\
\hline 4 & & & $<18$ & 7 & 180 & 5 & & 63 & 27 & -91 \\
\hline 5 & & & $<16$ & 13 & 180 & 6 & & 70 & 54 & 92 \\
\hline 6 & & & $<16$ & 3 & 0 & 7 & & $\mathbf{5 4}$ & 38 & 90 \\
\hline 7 & & & $<16$ & 5 & 0 & 8 & & $<\mathbf{3 3}$ & 12 & -103 \\
\hline 0 & 14 & & $<18$ & 8 & 180 & 1 & 6 & 45 & 35 & 88 \\
\hline 1 & & & $<18$ & 12 & 180 & 2 & & $<21$ & 31 & -98 \\
\hline 2 & & & $<18$ & 12 & 180 & 3 & & 91 & 76 & -89 \\
\hline 3 & & & $<18$ & 14 & 0 & 4 & & 58 & 47 & 92 \\
\hline 4 & & & $<18$ & 18 & 0 & 5 & & 28 & 29 & 91 \\
\hline l & 15 & & $<16$ & 4 & 0 & 6 & & 28 & 26 & -87 \\
\hline 2 & & & 45 & 46 & 180 & 7 & & $<\mathbf{3 0}$ & 16 & 97 \\
\hline 3 & & & 35 & 20 & 0 & 8 & & 33 & 11 & -88 \\
\hline 4 & & & $<16$ & 8 & 0 & 0 & 7 & 94 & 82 & -88 \\
\hline 5 & & & 34 & 29 & 180 & 1 & & 42 & 36 & -95 \\
\hline 0 & 16 & & $<16$ & 0 & 0 & 2 & & 24 & 27 & 88 \\
\hline 1 & & & 47 & 39 & 0 & 3 & & $<23$ & 10 & 112 \\
\hline 2 & 0 & 1 & 69 & 59 & 91 & 4 & & $<26$ & 19 & 94 \\
\hline 4 & & & 93 & 69 & 87 & 5 & & 40 & 26 & 90 \\
\hline 6 & & & 50 & 16 & 78 & 6 & & $<\mathbf{3 0}$ & 15 & -93 \\
\hline 8 & & & 73 & 40 & 91 & 7 & & 45 & 38 & -89 \\
\hline 1 & 1 & 1 & 87 & 55 & 90 & 1 & 8 & 26 & 24 & -85 \\
\hline 2 & & & 73 & 71 & 89 & 2 & & 23 & 10 & 103 \\
\hline 3 & & & $<19$ & 3 & 18 & 3 & & 56 & 48 & 92 \\
\hline 4 & & & $<26$ & 28 & 97 & 4 & & 34 & 23 & -95 \\
\hline 5 & & & $<23$ & 7 & -63 & 5 & & 50 & 30 & -93 \\
\hline 6 & & & 57 & 63 & 91 & 6 & & 30 & 4 & 96 \\
\hline 7 & & & $<28$ & 1 & 22 & 7 & & 33 & 10 & -85 \\
\hline 8 & & & $\mathbf{3 0}$ & 34 & -89 & 0 & 9 & $<\mathbf{3 0}$ & 19 & 87 \\
\hline 1 & 2 & & 20 & 38 & -79 & 1 & & 75 & 62 & 90 \\
\hline 2 & & & 126 & 101 & -87 & 2 & & $<26$ & 5 & -93 \\
\hline 3 & & & 20 & 14 & -100 & 3 & & $<28$ & 2 & -127 \\
\hline 4 & & & 80 & 79 & 88 & 4 & & $<28$ & 6 & -102 \\
\hline 5 & & & 71 & 42 & 94 & 5 & & 42 & 34 & -94 \\
\hline 6 & & & $<28$ & 12 & -90 & 6 & & $<\mathbf{3 0}$ & 8 & 88 \\
\hline 7 & & & $<28$ & 8 & 90 & 7 & & 45 & 29 & 91 \\
\hline 8 & & & $<30$ & 25 & -91 & 1 & 10 & $\mathbf{3 4}$ & 25 & 93 \\
\hline 0 & 3 & & & 106 & -86 & 2 & & $<28$ & 16 & -88 \\
\hline 1 & & & 41 & 93 & -87 & 3 & & 49 & 40 & -90 \\
\hline 2 & & & 41 & 38 & 86 & 4 & & $<30$ & 3 & 122 \\
\hline 3 & & & $<19$ & 5 & 17 & 5 & & $\mathbf{3 3}$ & 27 & 88 \\
\hline 4 & & & $<21$ & 13 & 97 & 6 & & $<\mathbf{3 3}$ & 7 & 105 \\
\hline 5 & & & & 27 & 92 & 0 & 11 & 58 & 36 & 95 \\
\hline 6 & & & 54 & 42 & -92 & 1 & & 65 & 48 & -90 \\
\hline 7 & & & $<28$ & 13 & -95 & 2 & & $<28$ & 1 & 172 \\
\hline 8 & & & 55 & 47 & 91 & 3 & & $<\mathbf{3 0}$ & 13 & 92 \\
\hline 1 & 4 & & 19 & 49 & -88 & 4 & & $<\mathbf{3 0}$ & 11 & -80 \\
\hline 2 & & & 105 & 85 & 88 & 5 & & $\mathbf{3 3}$ & 25 & 92 \\
\hline 3 & & & 42 & 41 & 86 & 6 & & $<\mathbf{3 0}$ & 12 & -86 \\
\hline 4 & & & 70 & 74 & -90 & 1 & 12 & $<30$ & 17 & -98 \\
\hline 5 & & & $<26$ & 23 & -91 & 2 & & $<30$ & 10 & 97 \\
\hline 6 & 4 & 1 & 34 & 26 & 93 & 3 & & 55 & 49 & 89 \\
\hline
\end{tabular}

Acta Chem. Scand. 18 (1964) No. 2 


\begin{tabular}{|c|c|c|c|c|c|c|c|c|c|}
\hline 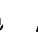 & $c$ & $\left|F_{0}\right|$ & $\left|\boldsymbol{F}_{\mathrm{c}}\right|$ & $a^{\circ}$ & $h$ & $k$ & $\left|F_{0}\right|$ & $\left|\boldsymbol{F}_{\mathrm{c}}\right|$ & $a^{\circ}$ \\
\hline 5 & & 35 & 32 & -87 & 0 & 6 & 35 & 35 & 177 \\
\hline 2 & 0 & 90 & 84 & 176 & 1 & & 71 & 77 & -177 \\
\hline 4 & & 51 & 16 & -29 & 2 & & 18 & 22 & -6 \\
\hline 6 & & 110 & 67 & $-\quad 3$ & 3 & & 34 & 27 & -168 \\
\hline 8 & & 125 & 66 & 180 & 4 & & 22 & 4 & -125 \\
\hline 1 & 1 & 111 & 101 & 7 & 5 & & 38 & 38 & $-\quad 2$ \\
\hline 2 & & 119 & 113 & 178 & 6 & & 51 & 32 & 176 \\
\hline 3 & & 46 & 34 & -177 & 7 & & $<28$ & 1 & 139 \\
\hline 4 & & 58 & 64 & -2 & 8 & & 32 & 35 & 6 \\
\hline 5 & & 79 & 68 & -176 & 1 & 7 & 44 & 36 & -179 \\
\hline 6 & & $<26$ & 6 & -138 & 2 & & 87 & 86 & 0 \\
\hline 7 & & $<26$ & 4 & $-\mathbf{3 0}$ & 3 & & $\mathbf{3 7}$ & 53 & -4 \\
\hline 8 & & $<28$ & 5 & 172 & 4 & & $<23$ & 4 & -146 \\
\hline 0 & 2 & 91 & 86 & 178 & 5 & & 45 & 40 & 180 \\
\hline 1 & & 64 & 45 & 4 & 6 & & 47 & 36 & -177 \\
\hline 2 & & 72 & 72 & $\mathbf{3}$ & 7 & & $<28$ & 14 & 18 \\
\hline 3 & & 100 & 82 & - & 0 & 8 & 47 & 31 & -173 \\
\hline 4 & & $<21$ & 16 & 4 & 1 & & 47 & 51 & 3 \\
\hline 5 & & $<21$ & 8 & -131 & 2 & & 44 & 42 & -176 \\
\hline 6 & & 59 & 61 & -177 & 3 & & 41 & 34 & 176 \\
\hline 7 & & 53 & 67 & -178 & 4 & & 44 & 41 & 1 \\
\hline 8 & & $<28$ & 28 & -6 & 5 & & 26 & 26 & -174 \\
\hline 1 & 3 & 37 & 35 & -176 & 6 & & 47 & 49 & 3 \\
\hline 2 & & 41 & 36 & -6 & 7 & & 47 & 50 & - \\
\hline 3 & & 38 & 42 & 7 & 1 & 9 & 45 & 39 & -8 \\
\hline 4 & & 80 & 84 & -177 & 2 & & $<23$ & 18 & 169 \\
\hline 5 & & $<23$ & 13 & 8 & 3 & & 61 & 63 & 179 \\
\hline 6 & & 49 & 45 & $-\quad 3$ & 4 & & $<26$ & 12 & 3 \\
\hline 7 & & $<26$ & 19 & -168 & 5 & & $<28$ & 9 & 23 \\
\hline 8 & & 32 & 36 & 2 & 6 & & $<28$ & 6 & -163 \\
\hline 0 & 4 & 152 & 124 & - & 7 & & $<28$ & 10 & 6 \\
\hline 1 & & 51 & 58 & -7 & 0 & 10 & 100 & 65 & -174 \\
\hline 2 & & $<17$ & 13 & 178 & 1 & & 43 & 37 & 180 \\
\hline 3 & & 36 & 29 & -18 & 2 & & $<23$ & 4 & -156 \\
\hline 4 & & 72 & 69 & 177 & 3 & & 26 & 34 & 2 \\
\hline 5 & & 38 & 38 & -177 & 4 & & $<26$ & 37 & 6 \\
\hline 6 & & $<26$ & 16 & -1 & 5 & & $<28$ & 20 & 5 \\
\hline 7 & & $<26$ & 5 & 17 & 6 & & $<28$ & 17 & 6 \\
\hline 8 & & $<28$ & 3 & -136 & 7 & 10 & 46 & 51 & 180 \\
\hline 1 & 5 & 36 & 28 & -177 & 1 & 11 & 26 & 29 & -175 \\
\hline 2 & & $<17$ & 18 & -143 & 2 & & 46 & 43 & -179 \\
\hline 3 & & 33 & 30 & -178 & 3 & & 47 & 42 & 1 \\
\hline 4 & & 51 & 62 & -4 & 5 & & $<28$ & 17 & 174 \\
\hline 5 & & 75 & 81 & 0 & 6 & & 47 & 39 & 5 \\
\hline 6 & & 25 & 28 & 176 & 1 & 12 & & 42 & - \\
\hline 7 & & $<28$ & 1 & 59 & 2 & & $<28$ & 8 & 3 \\
\hline 8 & & 28 & 34 & 178 & 3 & & $<28$ & 14 & 4 \\
\hline
\end{tabular}

The aim of the present structure investigation was to find out how the ether and monobromodichloromethane molecules are linked together. Even if earlier investigations seem to favour a hydrogen bond one could not rule out the possibility of a bond between the ether oxygen and one of the halogens in the solid state, as actually found in recent investigations by Hassel and coworkers $^{11}$ in similar complexes. 


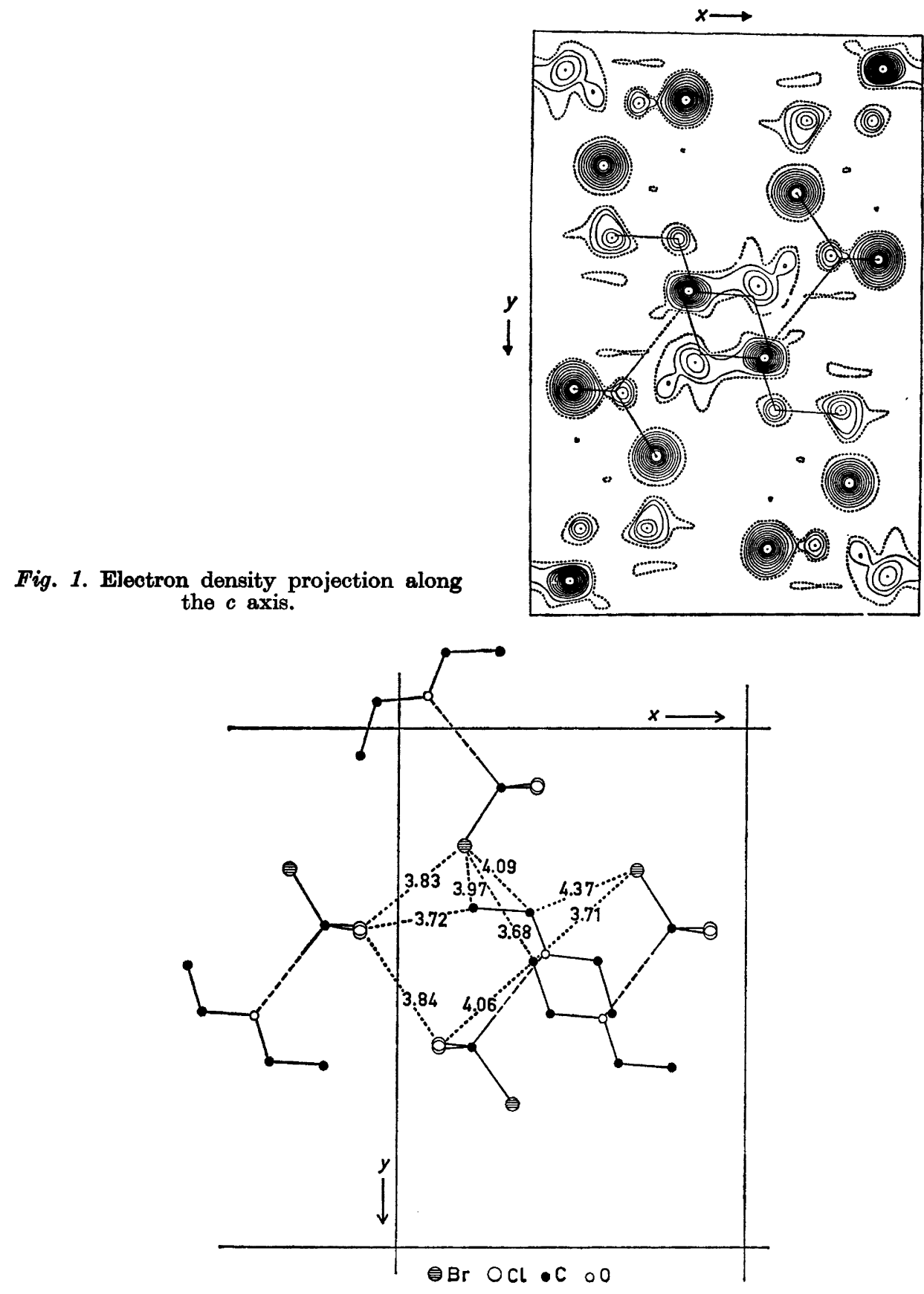

Fig. 2. The structure seen along the $c$ axis. Broken lines indicate the shortest intermolecular separations. (Pairs of chlorine atoms, actually overlapping are drawn separately).

Acta Chem. Scand. 18 (1964) No. 2 
This structure determination clearly shows that the formation of the ethermonobromodichloromethane complex depends on a $\mathrm{C}-\mathrm{H}---\mathrm{O}$ bond. The $\mathrm{C}-\mathrm{H}-\ldots-\mathrm{O}$ hydrogen bond distance is found to be $3.10 \AA$ with a standard deviation of about $0.1 \AA$. This result is in good agreement with other investigations of similar hydrogen bond distances as can be seen from the survey by Sutor. ${ }^{12}$

The intermolecular distances may be found in Fig. 2. They are mostly in reasonable agreement with generally accepted values for van der Waals' distances. Some of the halogen methyl- and methylene separations are somewhat shorter than should be expected, however. As nothing can be stated with certainty regarding the positions of the hydrogen atoms in these groups, a discussion of the significance of the shortening is not possible.

Acknowledgement. The authors wish to thank Professor Odd Hassel for suggesting this investigation and for his interest in the work.

\section{REFERENCES}

1. Wyatt, W. F. Trans. Faraday Soc. 25 (1929) 50.

2. Macleod, D. B. and Wilson, F. J. Trans. Faraday Soc. 31 (1935) 596.

3. Hassel, O. and Uhl, A. H. Naturwiss. 18 (1930) 247; Z. physik. Chem. 8 (1930) 187.

4. Bushwell, A. M., Rodebush, W. H. and Roy, M, F.J. Am. Chem. Soc. 60 (1938) 2528.

5. Gordy, W. Nature 142 (1938) 831.

6. Terenin, A. V. and Yeroslavskij, N. Acta Physiochim. U.S.S.R. 17 (1942) 240.

7. Dadieu, A. and Kohlrausch, K. W. F. Physik. Z. 31 (1930) 514.

8. Lemonde, H. Compt. Rend. 202 (1936) 468.

9. Parshad, R. Indian J. Phys. 16 (1942) 307.

10. Glasstone, S. Trans. Faraday Soc. 33 (1937) 200.

11. Hassel, O. and Römming, Chr. Quart. Rev. (London)) 16 (1962) 1.

12. Sutor, D. J. J. Chem. Soc. 19631105. 